\title{
Analysis of Shared Genetic Regulatory Networks for Alzheimer's Disease and Epilepsy
}

\author{
Xiao-Dan Wang, Shuai Liu, Hui Lu, Yalin Guan, Hao Wu, and Yong Ji \\ Department of Neurology, Tianjin Huanhu Hospital, Tianjin Key Laboratory of Cerebrovascular and Neurodegenerative Diseases, \\ Tianjin Dementia Institute, Tianjin 300350, China
}

Correspondence should be addressed to Yong Ji; jiyongusa@126.com

Xiao-Dan Wang and Shuai Liu contributed equally to this work.

Received 14 December 2020; Accepted 31 August 2021; Published 14 October 2021

Academic Editor: Cynthia C. Cardoso

Copyright (c) 2021 Xiao-Dan Wang et al. This is an open access article distributed under the Creative Commons Attribution License, which permits unrestricted use, distribution, and reproduction in any medium, provided the original work is properly cited.

\begin{abstract}
Alzheimer's disease (AD) and epilepsy are neurological disorders that affect a large cohort of people worldwide. Although both of the two diseases could be influenced by genetic factors, the shared genetic mechanism underlying the pathogenesis of them is still unclear. In this study, we aimed to identify the shared genetic networks and corresponding hub genes for AD and epilepsy. Firstly, the gene coexpression modules (GCMs) were constructed by weighted gene coexpression network analysis (WGCNA), and 16 GCMs were identified. Through further integration of GCMs, genome-wide association studies (GWASs), and expression quantitative trait loci (eQTLs), 4 shared GCMs of $\mathrm{AD}$ and epilepsy were identified. Functional enrichment analysis was performed to analyze the shared biological processes of these GCMs and explore the functional overlaps between these two diseases. The results showed that the genes in shared GCMs were significantly enriched in nervous system-related pathways, such as Alzheimer's disease and neuroactive ligand-receptor interaction pathways. Furthermore, the hub genes of AD- and epilepsy-associated GCMs were captured by weighted key driver analysis (wKDA), including TRPC1, C2ORF40, NR3C1, KIAA0368, MMT00043109, STEAP1, MSX1, KL, and CLIC6. The shared GCMs and hub genes might provide novel therapeutic targets for $\mathrm{AD}$ and epilepsy.
\end{abstract}

\section{Introduction}

Alzheimer's disease $(\mathrm{AD})$ is a neurodegenerative disease characterized by memory difficulty, daily activity dysfunction, and cognitive decline, with neuropathological lesion and neuron loss in the brain [1-3]. This disease affects approximately 36 million people worldwide, and the number of $\mathrm{AD}$ patients is estimated to triple by 2050 , along with the prolonged life expectancy of global population $[4,5]$. Currently, the therapies of $\mathrm{AD}$ mainly focus on counterbalancing the neurotransmitter disturbance, and there have not been effective pharmacotherapeutic options for the prevention and treatment of $\mathrm{AD}$ yet $[6,7]$. Another neurological disorder, epilepsy, is a common brain condition with an unprovoked seizure of high recurrence rate, which is defined epilepsy based on at least one of the following conditions: (1) two unprovoked seizures that occur more than $24 \mathrm{~h}$ apart and (2) diagnosis of an epilepsy syndrome $[8,9]$. Epilepsy is known to be closely related to the psychosocial, neurobiological, and cognitive statuses and affects more than 70 million people worldwide $[8,10]$. Despite the advances in antiepileptic drugs and surgeries, epilepsy treatments still confront some challenges, such as the resistance to medical treatment, underutilization of epilepsy surgery, and the gaps in epilepsy-related knowledge [11]. Moreover, the etiologies of $\mathrm{AD}$ and epilepsy are still unclear $[12,13]$.

It is believed that there is an association between $\mathrm{AD}$ and epilepsy [14]. Both AD and epilepsy are neurological diseases [15], and their incidence risks are proven to elevate with age. $\mathrm{AD}$ is known to influence more than $40 \%$ of the people over the age of 85 [16], and the incidence rate of epilepsy also remains high in patients over 50 years old and 
peaks at the age of 70 years [10]. In addition, accumulating evidence has proven that $\mathrm{AD}$ contributes to higher risk of epilepsy [17-19]. It is reckoned that $10-22 \%$ of $\mathrm{AD}$ patients have epilepsy [20]. Pathological alterations in entorhinal cortex, subiculum, and hippocampal field CA1 are observed in both temporal lobe epilepsy and $\operatorname{AD}[18,21,22]$. Furthermore, both $\mathrm{AD}$ and epilepsy are associated with genetic factors. It has been proven that genetic factors play a pivotal role in the occurrence of $\mathrm{AD}$. In nonfamilial $\mathrm{AD}$, genetic factors have a predominant role and account for more than $60 \%$ of the cases; while in familial $\mathrm{AD}$, the familial genes in $\mathrm{AD}$ are autosomal dominant, often with point mutations in presenilin 1, presenilin 2, and amyloid precursor protein [23]. SCN1A is recognized as an epilepsy-related gene, and its variants are related to inherited genetic epilepsy [24]. Rohena et al. have proposed SNAP25 mutation as a risk factor for epilepsy, which could also lead to ataxia and seizure in animal model [25]. Scher et al. have reported MTHFR C677T variant as a potential genetic cause for epilepsy [26]. In addition, the shared genetic background of $\mathrm{AD}$ and epilepsy has also been explored. The presenilin 1 gene (PSEN1) mutations are demonstrated to be associated with $\mathrm{AD}$ and epilepsy. Epileptic seizures are recognized as the clinical phenotypes of PSEN1 AD, and PSEN1 AD is suggested to be considered as a genetic epilepsy syndrome according to the new International League Against Epilepsy nomenclature [27]. The genetic form of AD is characterized by aberrant amyloid- $\beta$ and potentially related to seizures [14]. Toral-Rios et al. have proposed that the changes in GSK $3 \beta$ and the encoding genes of tau are the genetic factors that contribute to $\mathrm{AD}$ and temporal lobe epilepsy developments [28].

However, the treatment of epilepsy in patients with $\mathrm{AD}$ remains to be a challenge, because the patients are prone to be affected by drug interactions and adverse effects [20]. If there is a close relationship between epilepsy and $\mathrm{AD}$, the therapeutic strategies may be shared. Therefore, the association between epilepsy and $\mathrm{AD}$ is a pivotal issue. In this research, we integrated the gene coexpression modules (GCMs), genome-wide association studies (GWASs), and expression quantitative trait loci (eQTLs) to identify the shared GCMs of AD and epilepsy. Then, the shared biological processes of the GCMs were investigated to analyze the functional overlaps between these two diseases through functional enrichment analysis. In addition, the hub genes of AD- and epilepsy-associated GCMs were captured by weighted key driver analysis (wKDA). The shared GCMs and hub genes may be novel therapeutic targets for both $\mathrm{AD}$ and epilepsy.

\section{Materials and Methods}

2.1. Study Population. The gene expression profiles of epilepsy and $\mathrm{AD}$ samples were obtained from the Gene Expression Omnibus (GEO, https://www.ncbi.nlm.nih.gov/geo/) database. The epilepsy samples (access number: GSE63808, platform: Illumina HumanHT-12 V3.0) included 129 epilepsy patients, and the $\mathrm{AD}$ samples (access number: GSE132903, platform: Illumina HumanHT-12 V4.0) con- sisted of $97 \mathrm{AD}$ patients and 98 healthy controls. The genome-wide association study (GWAS) dataset of AD samples was obtained from Database of Genotypes and Phenotypes (dbGaP, https://www.ncbi.nlm.nih.gov/gap, access number: phs000219).

2.2. Data Preprocessing. Due to the difference in platforms of GSE63808 and GSE132903 datasets, the annotated genes were different. The removeBatchEffect function in limma package (http://www.bioconductor.org/packages/release/ bioc/html/limma.html) [29] was used to remove the batch effects after obtaining the shared genes of the two platforms. Then, the two datasets were integrated into a matrix for further analysis.

2.3. Construction of Gene Coexpression Modules (GCMs). The AD (case) and epilepsy expression data were used together for the GCM identification. The control samples in the GSE132903 dataset were not included in this analysis. We performed the weighted gene coexpression network analysis (WGCNA) by using WGCNA R package [30]. First, the similarity matrix was constructed by calculating the Pearson correlation coefficient between every two genes with the following equation: $S_{i j}=\left|\left(1+\operatorname{cor}\left(x_{i}+y_{j}\right)\right) / 2\right|$. Then, the similarity matrix was transformed to adjacency matrix with the equation of $\alpha_{i j}=\left|\left(1+\operatorname{cor}\left(x_{i}+y_{j}\right)\right) / 2\right|^{\beta}$, and $\beta$ represented the soft-threshold. The topological matrix was obtained based on the topological overlap measure (TOM) $=\left(\sum_{\mu \neq i j} \alpha_{i \mu} \alpha_{\mu j}+\alpha_{i j}\right) /\left(\min \left(\sum_{\mu} \alpha_{i \mu}+\sum_{\mu} \alpha_{j \mu}\right)+1-\alpha_{i j}\right)$.

Hierarchical clustering analysis was carried out using 1TOM, the index which reflected the similarity between every two genes. The modules were identified with dynamic branch cutting method, and the closely interconnected genes were placed into the same module (minimum module size $=30$ ). Module eigengene (ME), the representative gene of each module that reflected the whole expression level of corresponding module, was calculated using the equation of $\mathrm{ME}=$ princomp $\left(x_{i j}{ }^{q}\right)$.

2.4. Identification of Shared GCMs between AD and Epilepsy. The Marker Set Enrichment Analysis (MSEA) in Mergeomics [31] was adopted to identify the shared GCMs between $\mathrm{AD}$ and epilepsy, and the parameters that were used followed the default pipeline stablished by Shu et al. $P$ value $=0.05$ was used as the threshold for screening, and the filtered expression quantitative trait loci (eQTLs) were analyzed by MSEA. Three files were input: the summary results of GWAS, the eQTL information of single nucleotide polymorphisms (SNPs), and the GCM results of WGCNA.

2.5. Functional Enrichment Analysis. The KOBAS 3.0 (http://kobas.cbi.pku.edu.cn/index.php) was used to perform the functional enrichment analysis of genes. $P<0.05$ was considered as the threshold to screen for significantly enriched Kyoto Encyclopedia of Genes and Genomes (KEGG) and Reactome pathways.

2.6. Identification of Hub Genes. Hub genes are defined as the genes with the highest degree (the number of genes 


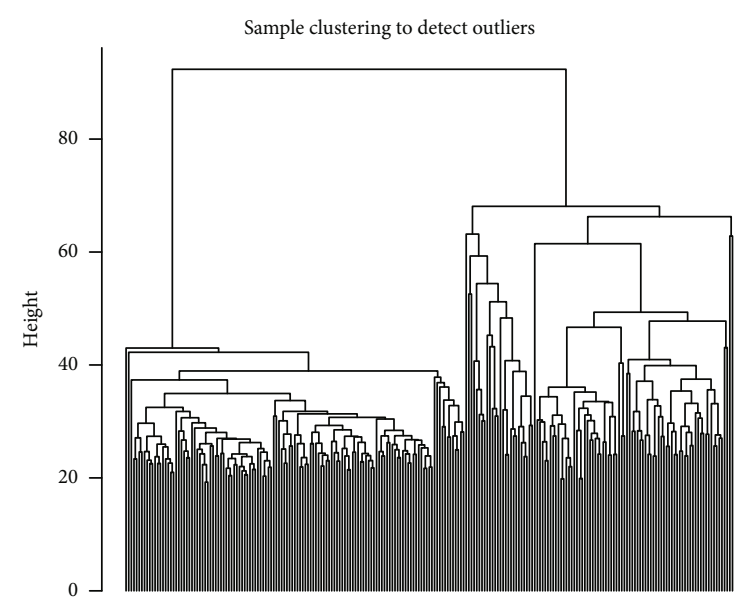

(a)
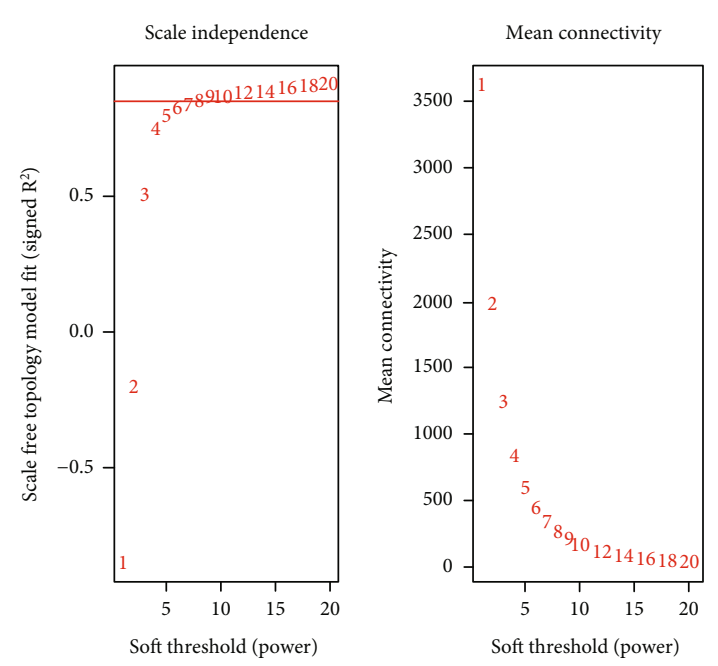

(b)

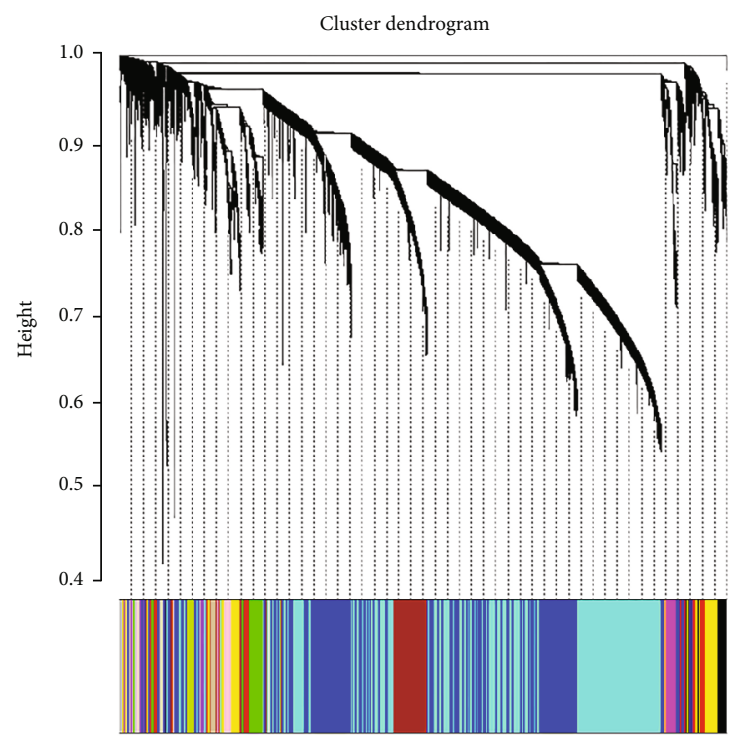

Module colors

(c)

FIGURE 1: Construction of gene coexpression modules. (a) Clustering analysis of the samples showed there were no outlier samples. (b) The soft-threshold was selected as $\beta=8$ to satisfy the criteria of scale free topology. (c) Gene dendrogram showed that 16 coexpression modules were identified. The gray module denoted the genes that could not be classified into any modules.

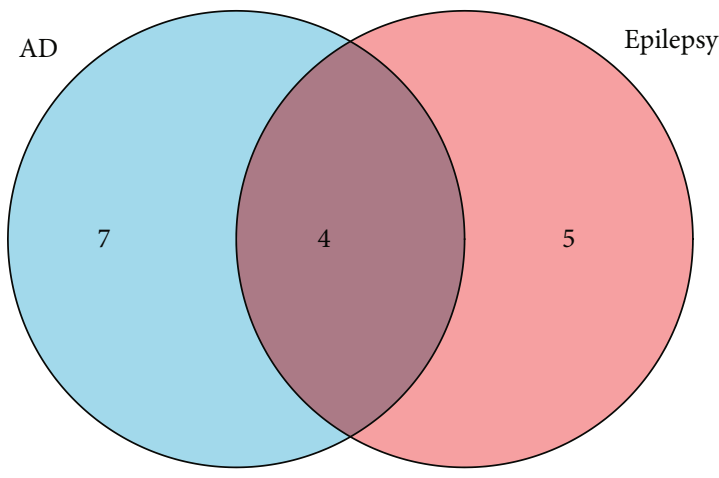

(a)

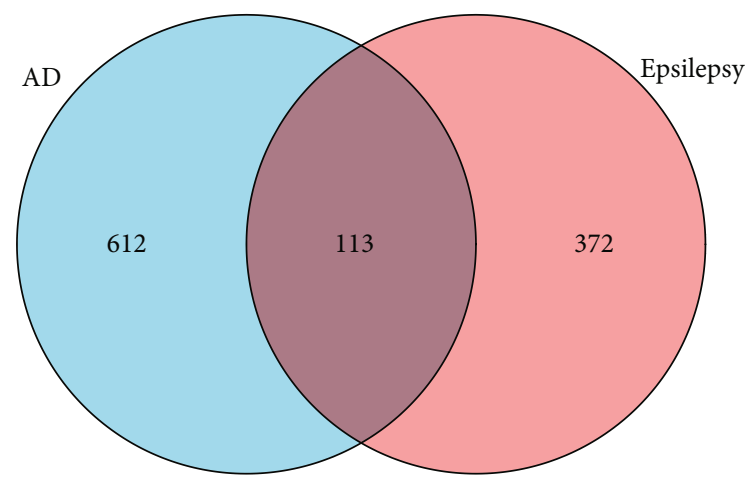

(b)

FIgURE 2: Venn diagrams of overlap in gene coexpression modules and pathways between AD and epilepsy. (a) Overlap in gene coexpression modules between $\mathrm{AD}$ and epilepsy. (b) Overlap in pathways between $\mathrm{AD}$ and epilepsy. 


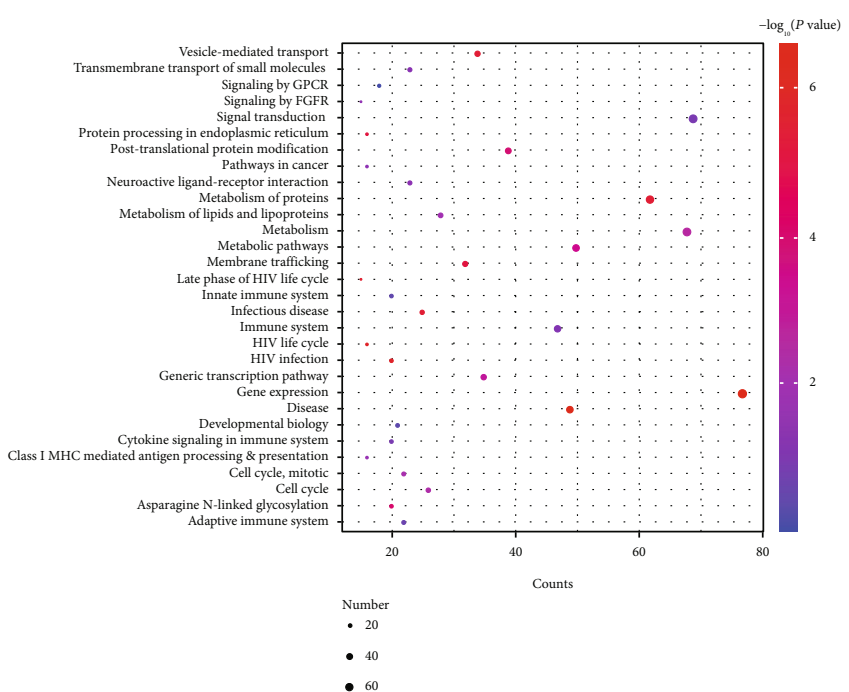

(a)

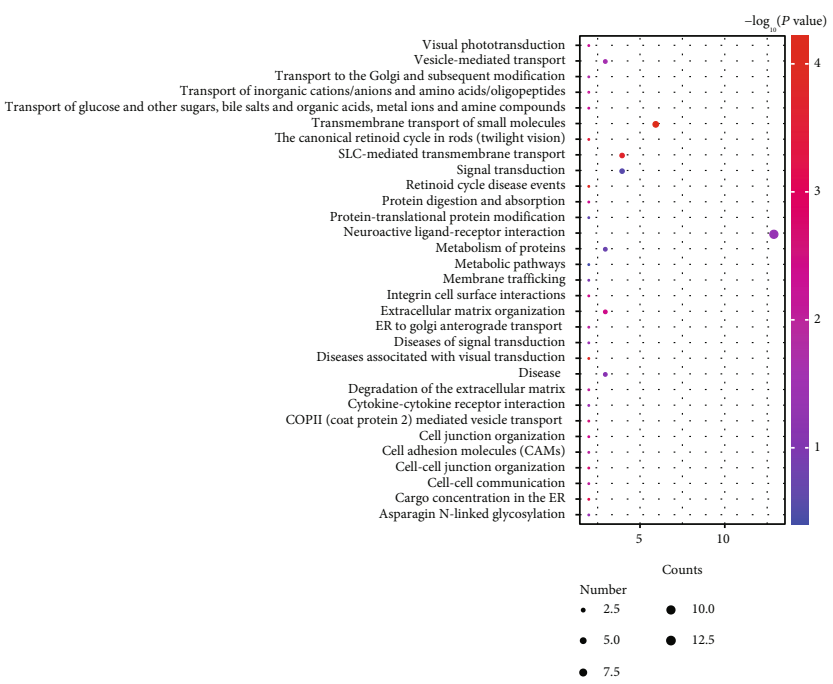

(b)

FIGURE 3: Function enrichment analysis of the genes in the (a) brown and (b) midnightblue modules. The vertical axis represented the pathways, and the color alteration of the dot from red to blue indicated the alteration of $P$ value from large to small.

connected to the hub) in the gene coexpression networks calculated by maximum neighborhood component (MNC) algorithm. The weighted key driver analysis (wKDA) in Mergeomics was used to identify the hub genes of each GCM, and the results were visualized by the Cytoscape software [32]. The above analyses were performed using the $\mathrm{R}$ software (version 3.5.2).

\section{Results}

3.1. A Total of 16 GCMs Were Identified for AD and Epilepsy Samples. There were 22,614 common genes for the gene expression profiles of $\mathrm{AD}$ and epilepsy samples. After removal of batch effects and integration of matrix, 22,614 genes were obtained. Clustering analysis showed that there were no outlier samples (Figure 1(a)). The soft-threshold was selected as $\beta=8$ to meet the criteria of scale free topology (the correlation coefficient between $\log (k)$ and $\log (p($ k)) greater than 0.8) (Figure 1(b)) $[33,34]$.

After identification of the gene modules, the ME of each module was calculated. Then, clustering analysis was performed on the modules, and the highly correlated modules were merged (height $=0.25$ ). As shown in Figure $1(\mathrm{c}), 16$ modules were obtained.

3.2. Shared GCMs between AD and Epilepsy. The identified 16 modules included AD-related modules, epilepsy-related modules, and modules associated with both AD and epilepsy. The shared GCMs between AD and epilepsy were identified by MSEA using the GCM information above and the GWAS data of AD. As shown in Figure 2(a), 11 modules were associated with $\mathrm{AD}$ and 9 with epilepsy. Among them, four modules (the brown, yellow, red, and midnightblue modules) were associated with both $\mathrm{AD}$ and epilepsy. Functional enrichment analysis of the genes in these 16 modules revealed that there were 725 pathways related to $\mathrm{AD}$ and 485 pathways related to epilepsy. Among them, 113 pathways were related to both AD and epilepsy (Figure 2(b)).

3.3. Shared Biological Processes of AD- and EpilepsyAssociated GCMs. Functional enrichment analysis of the genes in the 4 shared modules between $\mathrm{AD}$ and epilepsy was carried out. The significantly enriched pathways in the brown, midnightblue, red, and yellow modules are shown in Figures 3(a) and 3(b) and Figure S1A and Figure S1B, respectively. There were 713 genes in brown module, 152 in midnightblue module, 287 in red module, and 486 in yellow module. In addition, the result of functional enrichment analysis of these four modules was provided in Table S1. It was found that the genes in the 4 shared GCMs were significantly enriched in nervous systemrelated pathways, such as $\mathrm{AD}$ and neuroactive ligandreceptor interaction, indicating that the genes in these GCMs played a key role in $\mathrm{AD}$ and epilepsy.

3.4. Hub Genes of AD- and Epilepsy-Associated GCMs. The wKDA in Mergeomics was used to construct the gene expression networks for the 4 shared GCMs. Then, the hub genes were identified. As shown in Figure 4 and Figure S2, KIAA0368 in the brown module, MMT00043109 and TRPC1 in the red module, STEAP1, C2ORF40, MSX1, KL, and CLIC6 in the midnightblue module, and NR3C1 in the yellow module exhibited the highest degree in each of the gene coexpression networks and were identified as the hub genes of each module.

\section{Discussion}

As neurological disorders, $\mathrm{AD}$ and epilepsy are considered to be interconnected with shared symptoms and etiologies [35]. In addition, genetic factors also play a crucial role in the pathogenesis of $\mathrm{AD}$ and epilepsy. In this research, we 

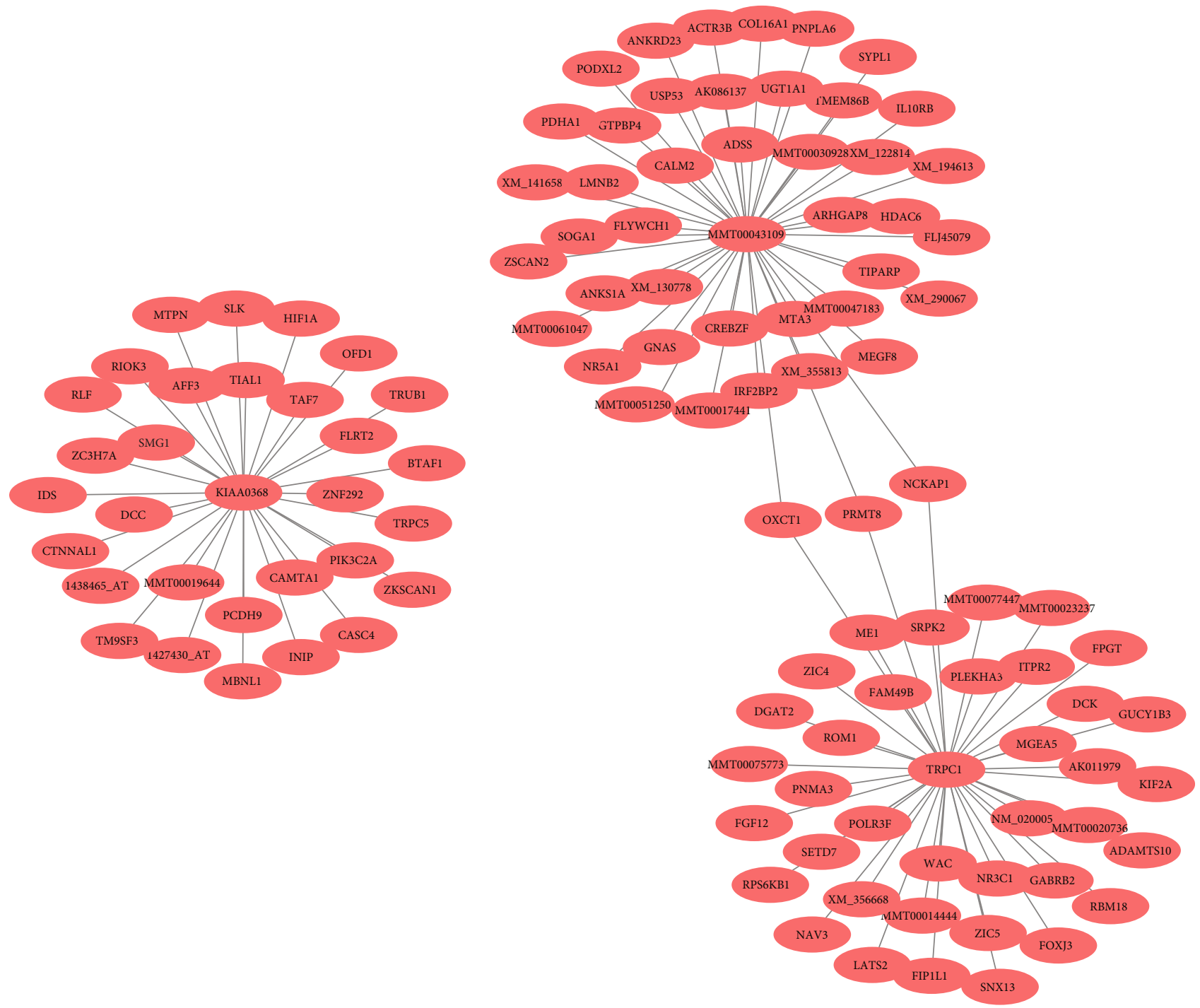

(a)

(b)

FIGURE 4: Identification of the hub genes for two of the 4 shared GCMs. (a) KIAA0368 was the hub gene of the brown module. (b) MMT00043109 and TRPC1 were the hub genes of the red module.

constructed 16 GCMs and identified 4 shared GCMs between $\mathrm{AD}$ and epilepsy by integrated analyses of GCMs, GWAS, and eQTLs. To analyze the functional overlaps between the two diseases, the shared biological processes of these GCMs were investigated by functional enrichment analysis. It revealed that the genes in the shared GCMs were significantly enriched in nervous system-related pathways, such as $\mathrm{AD}$ and neuroactive ligand-receptor interaction pathways. Furthermore, the hub genes of each AD- and epilepsy-associated GCM were captured, including TRPC1, C2ORF40, NR3C1, KIAA0368, MMT00043109, STEAP1, $M S X 1, K L$, and CLIC6.

Transient receptor potential canonical 1 (TRPC1) encodes the TRPC1 protein, which is a crucial member of the TRPC proteins [36]. TRPC proteins are main gates of $\mathrm{Ca}^{2+}$ entry and participate in multiple biological processes, including transcription factor activation and cell proliferation [37]. As first reported in 1995, TRPC1 protein can interact with TRPC4 and TRPC5 to form TRPC1/4/5 channels, which are significantly implicated in epilepsy [38-40]. It is generally known that TRPC1/4 channels can lead to the excitotoxicity and epileptiform burst firing in the CA1 and the lateral septum, which are pathophysiological elements of epilepsy [41, 42]. Consequently, TRPC1 is the potential target for the treatment of epilepsy. Previous study has suggested that calcium homeostasis dysfunction could occur in AD [43]. Due to the vital effect of TRPC1 on $\mathrm{Ca}^{2+}$ entry, it is hypothesized that TRPC1 is also probably associated with AD. A previous study confirmed that TRPC1 played a protective role in neurodegeneration and neurotoxicity, which were typical features of $\mathrm{AD}$, through regulation of $\mathrm{Ca}^{2+}$ influx [44-46]. Therefore, TRPC1 may be a shared genetic risk factor for $\mathrm{AD}$ and epilepsy.

The orphan C2ORF40 gene encodes Ecrg4, a preprotein precursor related to neural progenitor responsiveness of central nervous system injury [47, 48]. The expression of 
C2ORF40 alters in some brain cells of AD patients, which is probably implicated in the neuroimmune response in $\mathrm{AD}$ via recruiting the microglia or infiltrating monocytes to the white matter [49], while in status epilepticus, the infiltrating monocytes could enhance the inflammation of brain and accelerate neural injury [50]. Based on these studies, we speculate that C2ORF40 may also be involved in epilepsy by affecting the infiltrating monocytes.

NR3C1 encodes glucocorticoid receptor (GR), a $94 \mathrm{kDa}$ protein that belongs to the superfamily of nuclear hormone receptors [51]. GR plays an important role in neuron function by binding to glucocorticoid hormones [52]. In the mouse model of $\mathrm{AD}$, the early downregulation of GR was observed, and the decreased GR level could be normalized by rosiglitazone, a potent agonist used for the cognitive function improvement for $\mathrm{AD}$ patients [53]. Brain-derived neurotrophic factor $(B D N F)$, highly expressed in the brain, is a crucial regulator of neuron function, and the altered expression of $B D N F$ is related to epilepsy, while GR shares several similarities with $B D N F$ and is able to regulate $B D N F$ expression level [52]. Therefore, it is proposed that the GRencoding gene $N R 3 C 1$ may participate in the pathogenesis of epilepsy by regulating $B D N F$, and $N R 3 C 1$ is a possible shared gene for $\mathrm{AD}$ and epilepsy.

In conclusion, we identified 4 shared GCMs and 9 hub genes, including TRPC1, C2ORF40, NR3C1, KIAA0368, MMT00043109, STEAP1, MSX1, KL, and CLIC6 through integrating analyses of genomics and genetics. The shared GCMs and hub genes should be helpful for unraveling the current difficulty of epilepsy treatment in $\mathrm{AD}$ and providing novel shared therapeutic strategies targeting both of these two diseases.

\section{Data Availability}

All data generated or analyzed during this study are included in this published article.

\section{Conflicts of Interest}

The authors declare that they have no competing interests, and all authors should confirm its accuracy.

\section{Authors' Contributions}

Xiao-Dan Wang contributed to the methodology, formal analysis, software, and writing original draft; Shuai Liu contributed to the methodology and visualization; HL contributed to the supervision; YLG and HW contributed to the writing-review and editing. Yong Ji contributed to the investigation, supervision, conceptualization, funding acquisition, and project administration. Xiao-Dan Wang and Shuai Liu shared equal contribution.

\section{Acknowledgments}

This study was supported by the Science and Technology Foundation of Tianjin Municipal Health and Health Committee (grant number ZC20121) and Science and Technol- ogy Personnel Training Program of Tianjin Municipal Health and Health Committee (grant number KJ20048).

\section{Supplementary Materials}

Supplementary 1. Figure S1: function enrichment analysis of the genes in the red (A) and yellow modules (B). The vertical axis represented the pathways, and the color alteration of the dot from red to blue indicated the alteration of $P$ value from large to small.

Supplementary 2. Figure S2: identification of the hub genes for another two of the 4 shared GCMs. (A) STEAP1, C2ORF40, MSX1, KL, and CLIC6 were the hub genes of the midnightblue module. (B) NR3C1 was the hub gene of the yellow module.

Supplementary 3. Table S1: functional enrichment analysis of these four modules.

\section{References}

[1] H. Keren-Shaul, A. Spinrad, A. Weiner et al., "A unique microglia type associated with restricting development of Alzheimer's disease," Cell, vol. 169, no. 7, pp. 1276-1290.e17, 2017.

[2] W. A. Kukull and J. D. Bowen, "Dementia epidemiology," Medical Clinics of North America, vol. 86, no. 3, pp. 573-590, 2002.

[3] S. T. DeKosky and K. Marek, "Looking backward to move forward: early detection of neurodegenerative disorders," Science, vol. 302, no. 5646, pp. 830-834, 2003.

[4] R. Balez and L. Ooi, "Getting to NO Alzheimer's Disease: Neuroprotection versus Neurotoxicity Mediated by Nitric Oxide," Oxidative Medicine and Cellular Longevity, vol. 2016, Article ID 3806157, 8 pages, 2016.

[5] A. Ritter and J. Cummings, "Fluid biomarkers in clinical trials of Alzheimer's disease therapeutics," Frontiers in Neurology, vol. 6, p. 186, 2015.

[6] K. G. Yiannopoulou and S. G. Papageorgiou, "Current and future treatments for Alzheimer's disease," Therapeutic Advances in Neurological Disorders, vol. 14, pp. 4-7, 2009.

[7] J. P. Michaud, M. Halle, A. Lampron et al., "Toll-like receptor 4 stimulation with the detoxified ligand monophosphoryl lipid a improves Alzheimer's disease-related pathology," Proceedings of the National Academy of Sciences of the United States of America, vol. 110, no. 5, pp. 1941-1946, 2013.

[8] R. S. Fisher, C. Acevedo, A. Arzimanoglou et al., "ILAE official report: a practical clinical definition of epilepsy," Epilepsia, vol. 55, no. 4, pp. 475-482, 2014.

[9] E. Beghi, G. Giussani, and J. W. Sander, "The natural history and prognosis of epilepsy," Epileptic Disorders, vol. 17, no. 3, pp. 243-253, 2015.

[10] R. D. Thijs, R. Surges, T. J. O'Brien, and J. W. Sander, "Epilepsy in adults," The Lancet, vol. 393, no. 10172, pp. 689-701, 2019.

[11] S. L. Moshé, E. Perucca, P. Ryvlin, and T. Tomson, "Epilepsy: new advances," The Lancet, vol. 385, no. 9971, pp. 884-898, 2015.

[12] T. Jiang, J. T. Yu, Y. Tian, and L. Tan, "Epidemiology and etiology of Alzheimer's disease: from genetic to non- genetic factors," Current Alzheimer Research, vol. 10, no. 8, pp. 852-867, 2013. 
[13] D. Sritharan and S. V. Sarma, "Fragility in dynamic networks: application to neural networks in the epileptic cortex," Neural Computation, vol. 26, no. 10, pp. 2294-2327, 2014.

[14] D. Friedman, L. S. Honig, and N. Scarmeas, "Seizures and epilepsy in Alzheimer's disease," CNS Neuroscience \& Therapeutics, vol. 18, no. 4, pp. 285-294, 2012.

[15] A. I. Kaplin, M. Williams, D. G. Hirtz, D. J. Thurman, K. Gwinn-Hardy, and R. Zalutsky, "How common are the "common" neurologic disorders?," Neurology, vol. 69, no. 4, pp. 410-411, 2007.

[16] K. Y. Yoo and S. Y. Park, "Terpenoids as potential anti-Alzheimer's disease therapeutics," Molecules, vol. 17, no. 3, pp. 35243538, 2012.

[17] S. Jenssen and D. Schere, "Treatment and management of epilepsy in the elderly demented patient," American Journal of Alzheimer's Disease and Other Dementias, vol. 25, no. 1, pp. 18-26, 2010.

[18] S. Ziyatdinova, K. Gurevicius, N. Kutchiashvili et al., "Spontaneous epileptiform discharges in a mouse model of Alzheimer's disease are suppressed by antiepileptic drugs that block sodium channels," Epilepsy Research, vol. 94, no. 1-2, pp. 7585, 2011.

[19] C. Hommet, R. Hureaux, J. Barré, T. Constans, and G. Berrut, "Epileptic seizures in clinically diagnosed Alzheimer's disease: report from a geriatric medicine population," Aging Clinical \& Experimental Research, vol. 19, no. 5, pp. 430-431, 2007.

[20] D. . C. Miranda and S. M. D. Brucki, "Epilepsy in patients with Alzheimer's disease: a systematic review," Dementia \& Neuropsychologia, vol. 8, no. 1, pp. 66-71, 2014.

[21] J. L. Price, A. I. Ko, M. J. Wade, S. K. Tsou, D. W. McKeel, and J. C. Morris, "Neuron number in the entorhinal cortex and cal in preclinical Alzheimer disease," Archives of Neurology, vol. 58, no. 9, pp. 1395-1402, 2001.

[22] H. E. Scharfman, "The neurobiology of epilepsy," Current Neurology \& Neuroscience Reports, vol. 7, no. 4, pp. 348-354, 2007.

[23] J. W. Ashford and J. A. Mortimer, "Non-familial Alzheimer's disease is mainly due to genetic factors," Journal of Alzheimers Disease, vol. 4, no. 3, pp. 169-177, 2002.

[24] D. Lal, E. M. Reinthaler, B. Dejanovic et al., "Evaluation of presumably disease causing SCN1a variants in a cohort of common epilepsy syndromes," PLoS One, vol. 11, no. 3, article e0150426, 2016.

[25] L. Rohena, J. Neidich, M. Truitt Cho et al., "Mutation in SNAP25 as a novel genetic cause of epilepsy and intellectual disability," Rare Diseases, vol. 1, no. 1, article e26314, 2013.

[26] A. I. Scher, H. Wu, J. W. Tsao et al., "MTHFR C677t genotype as a risk factor for epilepsy including post-traumatic epilepsy in a representative military cohort," Journal of Neurotrauma, vol. 28, no. 9, pp. 1739-1745, 2011.

[27] A. J. Larner, "Presenilin-1 mutation Alzheimer's disease: a genetic epilepsy syndrome?," Epilepsy \& Behavior, vol. 21, no. 1, pp. 20-22, 2011.

[28] D. Toral-Rios, P. S. Pichardo-Rojas, M. Alonso-Vanegas, and V. Campos-Peña, "GSK3 $\beta$ and tau protein in Alzheimer's disease and epilepsy," Frontiers in Cellular Neuroscience, vol. 14, p. 19, 2020.

[29] M. E. Ritchie, B. Phipson, D. Wu et al., "limma powers differential expression analyses for RNA-sequencing and microarray studies," Nucleic Acids Research, vol. 43, no. 7, p. e47, 2015.
[30] P. Langfelder and S. Horvath, "WGCNA: an R package for weighted correlation network analysis," BMC Bioinformatics, vol. 9, no. 1, p. 559, 2008.

[31] L. Shu, Y. Zhao, Z. Kurt et al., "Mergeomics: multidimensional data integration to identify pathogenic perturbations to biological systems,” BMC Genomics, vol. 17, no. 1, p. 874, 2016.

[32] P. Shannon, A. Markiel, O. Ozier et al., "Cytoscape: a software environment for integrated models of biomolecular interaction networks," Genome Research, vol. 13, no. 11, pp. 24982504, 2003.

[33] J. du, S. Wang, C. He, B. Zhou, Y. L. Ruan, and H. Shou, "Identification of regulatory networks and hub genes controlling soybean seed set and size using RNA sequencing analysis," Journal of Experimental Botany, vol. 68, no. 8, pp. 19551972, 2017.

[34] K. Greenham, C. R. Guadagno, M. A. Gehan et al., “Temporal network analysis identifies early physiological and transcriptomic indicators of mild drought in Brassica rapa," eLife, vol. 6, article e29655, 2017.

[35] N. Garg, R. Joshi, and B. Medhi, "Cracking novel shared targets between epilepsy and Alzheimer's disease: need of the hour," Reviews in the Neurosciences, vol. 29, no. 4, pp. 425-442, 2018.

[36] H. N. Rubaiy, M. J. Ludlow, M. Henrot et al., "TRPC1/4/5 inhibitor," Journal of Biological Chemistry, vol. 292, no. 20, pp. 8158-8173, 2017.

[37] J. Abramowitz and L. Birnbaumer, "Physiology and pathophysiology of canonical transient receptor potential channels," The FASEB Journal, vol. 23, no. 2, pp. 297-328, 2009.

[38] C. Strübing, G. Krapivinsky, L. Krapivinsky, and D. E. Clapham, "TRPC1 and TRPC5 form a novel cation channel in mammalian brain," Neuron, vol. 29, no. 3, pp. 645-655, 2001.

[39] Y. Akbulut, H. J. Gaunt, K. Muraki et al., "Englerin A is a potent and selective activator of TRPC4 and TRPC5 calcium channels," Angewandte Chemie International Edition, vol. 54, no. 12, pp. 3787-3791, 2015.

[40] S. Z. Xu, P. Sukumar, F. Zeng et al., "TRPC channel activation by extracellular thioredoxin," Nature, vol. 451, no. 7174, pp. 69-72, 2008.

[41] F. Zheng, "TRPC channels and epilepsy," Advances in Experimental Medicine and Biology, vol. 976, pp. 123-135, 2017.

[42] F. Zheng and K. Phelan, "The role of canonical transient receptor potential channels in seizure and excitotoxicity," Cell, vol. 3, no. 2, pp. 288-303, 2014.

[43] F. M. Laferla, "Calcium dyshomeostasis and intracellular signalling in Alzheimer's disease," Nature Reviews Neuroscience, vol. 3, no. 11, pp. 862-872, 2002.

[44] S. Yamamoto, T. Wajima, Y. Hara, M. Nishida, and Y. Mori, "Transient receptor potential channels in Alzheimer's disease," BBA-Molecular Basis of Disease, vol. 1772, no. 8, pp. 958-967, 2007.

[45] E. Popugaeva, O. L. Vlasova, and I. Bezprozvanny, "Restoring calcium homeostasis to treat Alzheimer's disease: a future perspective," Neurodegenerative Disease Management, vol. 5, no. 5, pp. 395-398, 2015.

[46] S. Varadarajan, S. Yatin, M. Aksenova, and D. A. Butterfield, "Review: Alzheimer's amyloid $\beta$-peptide-associated free radical oxidative stress and neurotoxicity," Journal of Structural Biology, vol. 130, no. 2-3, pp. 184-208, 2000.

[47] T. Su, H. Liu, and S. Lu, "Cloning and identification of cDNA fragments related to human esophageal cancer," Zhonghua Zhong Liu Za Zhi, vol. 20, no. 4, pp. 254-257, 1998. 
[48] S. Podvin, M. Miller, R. Rossi et al., "P2-023: White matter injury and the orphan c2orf40 gene encoding Ecrg4 in Alzheimer's disease," Alzheimerr's \& Dementia, vol. 9, pp. P352P353, 2013.

[49] S. Podvin, M. C. Miller, R. Rossi et al., "The orphan C2orf40 gene is a neuroimmune factor in Alzheimer's disease," JSM Alzheimer's Disease and Related Dementia, vol. 3, p. 1020, 2016.

[50] N. H. Varvel, J. J. Neher, A. Bosch et al., "Infiltrating monocytes promote brain inflammation and exacerbate neuronal damage after status epilepticus," Proceedings of the National Academy of Sciences, vol. 113, no. 38, pp. E5665-E5674, 2016.

[51] N. Niu, V. Manickam, K. R. Kalari et al., "Human glucocorticoid receptor alpha gene (NR3C1) pharmacogenomics: gene resequencing and functional genomics," The Journal of Clinical Endocrinology and Metabolism, vol. 94, no. 8, pp. 30723084, 2009.

[52] H. Chen, M. Lombès, and D. le Menuet, "Glucocorticoid receptor represses brain-derived neurotrophic factor expression in neuron-like cells," Molecular Brain, vol. 10, no. 1, p. 12, 2017.

[53] L. Escribano, A. M. Simón, A. Pérez-Mediavilla, P. SalazarColocho, J. D. Río, and D. Frechilla, "Rosiglitazone reverses memory decline and hippocampal glucocorticoid receptor down-regulation in an Alzheimer's disease mouse model," Biochemical and Biophysical Research Communications, vol. 379, no. 2, pp. 406-410, 2009. 